Methods A retrospective analysis of case report forms of 140 (65 asthma and 75 COPD) patients who underwent research bronchoscopy at our centre since November 2010.

Results See Table 1 for details.

Baseline characteristics were the same among COPD and asthma patients who did and did not receive bronchodilators. There was no significant change in procedure tolerance, sedation used, complications or adverse events and samples obtained in patients who received pre-procedure bronchodilators. Mean volume of saline inserted for BAL during bronchoscopy was $414.3 \pm 140.5 \mathrm{ml}$ for asthmatics and $392.1 \pm 123.5 \mathrm{ml}$ for COPD patients.

Overall, serious complications were rare: 2 patients bled during the procedure requiring cold saline and adrenaline, 1 was observed for a few hours due to low saturations, 1 was admitted overnight for hypotension and 1 was admitted with pleuritic chest pain.

41 patients were symptom free at 24 hours and 85 were symptom free at 7 days. The most common mild symptom reported at 24 hours was sore throat, being reported by 50 patients; at 7 days 21 patients reported cough.

Conclusion Nebulised bronchodilators pre-bronchoscopy in patients with asthma or COPD appears to have little impact. Overall, research bronchoscopy with significant BAL in these patients appears relatively safe.

* The first 2 authors contributed equally to this work.

\section{M16 STAFF-PATIENT PERCEPTION OF DISCOMFORT WITH FIBRE-OPTIC BRONCHOSCOPY-IS THERE A CORRELATION?}

LO Idris, V Richardson, I Johnson, G Sobrany, KS Babu; Queen Alexandra Hospital, Portsmouth, United Kingdom

\subsection{6/thoraxjnl-2013-204457.426}

Introduction Patient comfort and safety are crucial aspects of fiberoptic bronchoscopy. This is usually performed under sedation and at times it is tricky to judge the degree of sedation and patient comfort during the procedure.

Methods We conducted a prospective survey to assess patients' satisfaction with sedation and the overall experience during flexible bronchoscopy. This was a questionnaire based survey, wherein the patients' completed a questionnaire within 48 hours after the procedure. We advised them not to complete the questionnaire on the same day of the procedure to avoid bias due to the effects of sedatives used during procedure. The questions were scored on a 5-point Likert scale. The questionnaire included satisfaction regarding the procedure, staff professionalism, the endoscopy suite, perception of adequacy of sedation, technical ability of the interventionalist, and post procedure care. Nursing staff were requested to record their perception of the degree of sedation and patient discomfort. Correlation between patients perception of discomfort/pain were compared with the staff perception.

Results 52 patients completed the questionnaire over a 3 month period. 33/52 (63.7\%) experienced pain/discomfort during the procedure. $73.1 \%$ felt sufficient steps were taken to reduce the pain/discomfort. $46(88.4 \%)$ of patients' disclosed that they did not mind to have a repeat procedure if needed. While there was poor correlation between the protocol of sedation used and patient comfort, there was a significant correlation between the staff perception of adequacy of sedation with the patients perception $(\mathrm{p}=0.0007)$.
Conclusion Regular patient surveys would give us an idea about the sedation practices we employ for bronchoscopy. As staffs perception significantly correlates with patients' pain/discomfort this can be a valuable tool in judging the sedation requirements especially in a partly sedated patient.

\section{M17 SURVEY ASSESSING METHODS OF VOCAL CORD ANAESTHESIA DURING BRONCHOSCOPY}

DA Tarpey; Southport and Ormskirk NHS trust, Liverpool, United Kingdom

\subsection{6/thoraxjnl-2013-204457.427}

Introduction To anaesthetise the vocal cords during bronchoscopy there are 2 methods in general use, transcricoid lidocaine or lidocaine administered directly through the bronchoscope (direct vision). The choice between the two methods is down to individual operator choice and there has been little work comparing each method. We performed a survey assessing several aspects of bronchoscopy to see if there was a difference between the 2 approaches.

Methods The bronchoscopist (one of three consultants and two registrars) and two nurses assessed patients degree of coughing, choking, sedation and overall tolerance of the procedure using a 10 -point visual analogue scale. All patients were given $2-4 \mathrm{mg}$ of midazolam as a sedative as is normal practice in our trust. Method of local anaesthesias and outcome of the bronchoscopy were noted. Results were analysed with a paired test.

Results 33 patients were assessed, 14 patients had direct vision lidocaine and 19 had transcricoid lidocaine. 2 of the procedures were abandoned due to patient's intolerance (both in the direct vision group). There was a significant reduction in coughing (3.5 vs. $5.7 \mathrm{p}$ value 0.009 ) choking (1.9 vs. 3.9 p 0.004$)$ and overall tolerance was better in the transcricoid group $(8.0$ vs. $5.6 \mathrm{p}$ $0.003)$. There was no difference in the degree of sedation ( 5.4 vs. $4.9 \mathrm{p} \mathrm{0.4)}$. There was no significant difference in the amount of successful biopsies performed in each group. There was no difference in the amount of midazolam given to each group $(2.65 \mathrm{mg}$ vs. $2.68 \mathrm{mg}$ ) and the differences were preserved despite the individual bronchoscopist.

Conclusions In this small pilot study The transcricoid group coughed and choked less and tolerated the procedure better in this survey. There was no difference between the groups in terms of sedation, total midazolam dosage or operator suggesting that this difference may due to the differing methods of local anaesthesia. A previous patient survey in our trust has shown patients themselves tolerated the transcricoid approach well. Further studies are needed to fully assess the differences between these two approaches and inform further practice.

\section{COPD: a clinical spectrum}

\section{M18 THE RELATIONSHIP BETWEEN ANXIETY AND DEPRESSION TO EXACERBATIONS OF COPD RESULTING IN HOSPITAL ADMISSIONS; A NARRATIVE SYSTEMATIC REVIEW}

${ }^{1}$ A Pooler, ${ }^{2} \mathrm{R}$ Beech; ${ }^{1}$ School of Nursing and Midwifery, Keele University, staffordshire, UK; ${ }^{2}$ Primary Care Sciences Research Institute, Keele University, staffordshire, UK

10.1136/thoraxjnl-2013-204457.428 
Introduction and Objectives Exacerbations because of COPD are the third largest cause of emergency hospital admissions in the UK. This systematic literature review explored the relationshipbetween hospitalisation rates and the COPD co-morbidities, anxiety and depression.

Methods The Centre for Research Dissemination's framework for systematic reviews was followed using search terms relating to COPD, anxiety, depression and hospital admission. Papers identified were assessed for relevance and quality using a suitable CASP tool and the Mixed Methods Assessment Tool (MMAT).

Results Quantitative studies (18) indicated that anxiety and depression led to a statistically significant increase in the likelihood of COPD patients being hospitalised. These co-morbidities also led to an increased length of stay and a greater risk of mortality post discharge. Other significant factors included lower BODE scores, female gender, lower socioeconomic status, poorer patient perceived quality of life, increased severity of lung function and less improvement in dyspnoea from admission to discharge. It was also highlighted that only $27-33 \%$ of those with depression were being treated for it. Qualitative studies (6), revealed that patients saw anxiety and depression as a major factor that affected their ability to cope with and self-manage their condition.

Implications Findings from the systematic review have highlighted a need for better recognition and treatment of anxiety and depression amongst individuals with COPD. On-going research will develop and test strategies for promoting better management and self-management as a means of reducing hospital admissions.

\section{M19 THE IMPACT OF CO-MORBIDITIES ON PHYSICAL FUNCTION AND HEALTH STATUS IN CHRONIC OBSTRUCTION PULMONARY DISEASE (COPD)}

NS Gale, AM Albarrati, IC Munnery, MM Munnery, JC Cockcroft, DJ Shale; Wales Heart Research Institute, Cardiff University, Cardiff, UK

\subsection{6/thoraxjnl-2013-204457.429}

Background Co-morbidities are of increasing importance in patients with COPD. However, the implications for function and health status have not been fully established. We hypothesised that the number of co-morbidities would relate to physical capacity, health status and impairments as measured by the comprehensive geriatric assessment (CGA) in COPD but not comparator subjects.

Method As part of the longitudinal Assessment of Risk in Chronic Airways Disease Evaluation (ARCADE), 500 patients with stable COPD (confirmed with spirometry) were compared to 141 comparator subjects (past or current smokers) free from respiratory disease. In all subjects previously diagnosed co-morbidities including; hypertension, hypercholesterolemia, angina, myocardial infarction, Stroke/TIA, atrial fibrillation, diabetes, and osteoporosis were recorded using a standardised health questionnaire. Spirometry, BMI, six minute walk distance (6MWD), the Timed Up and Go (TUG), and the number of impairments were determined using the CGA. Patients with COPD also completed the St George's Respiratory Questionnaire (SGRQ).

Results Patients and comparators were similar in age, gender and BMI, but differed in $\mathrm{FEV}_{1} \%$ predicted 59 (20) and 105 (14) respectively $(\mathrm{p}<0.01)$. Patients had more co-morbidities median (range) $2(0-6)$ than comparators $1(0-3)(\mathrm{p}<0.01)$. Of the patients, $24 \%$ had no co-morbidity, $54 \%$ had $1-2$ co-morbidities and $22 \%$ had over 3 co-morbidities, while $54 \%$ of comparators had no co-morbidities and 45\% had 1-2 co-morbidities ( $\mathrm{p}<$ 0.01). Patients also had more impairments (CGA score), reduced 6MWD and increased TUG (all $\mathrm{p}<0.001$ ). The number of comorbidities related to age, BMI, 6MWD, TUG, fibrinogen, the CGA and SGRQ and but not FEV 1 in patients with COPD, and only to CGA score in comparators (Table 1 ).

Conclusion The number of comorbidities in COPD related to physical function, health status and impairments, independent of lung disease. Early management of co-morbidities may improve outcomes in patients with COPD.

\begin{tabular}{|c|c|c|c|c|}
\hline & COPD & $p=$ & Comparator & $p=$ \\
\hline & $r_{s}$ & & $r_{s}$ & \\
\hline Age (years) & 0.209 & $<0.001$ & 0.119 & 0.161 \\
\hline $\mathrm{FEV}_{1} / \mathrm{FVC}(\mathrm{L})$ & -0.014 & 0.751 & 0.089 & 0.295 \\
\hline $\mathrm{FEV}_{1} \%$ predicted & -0.031 & 0.488 & 0.045 & 0.598 \\
\hline BMI $\left(\mathrm{kg} / \mathrm{m}^{2}\right)$ & 0.208 & $<0.001$ & 0.144 & 0.088 \\
\hline $6 \mathrm{MWD}(\mathrm{m})$ & -0.208 & $<0.001$ & -0.147 & 0.081 \\
\hline TUG (s) & 0.192 & $<0.001$ & -0.020 & 0.814 \\
\hline CGA score & 0.450 & $<0.001$ & 0.352 & $<0.001$ \\
\hline SGRQ total score & 0.147 & 0.001 & - & - \\
\hline
\end{tabular}

\section{M20 FINDING THE MISSING MILLIONS: CASE FINDING FOR COPD IN PEOPLE ATTENDING OTHER LONG TERM CONDITION CLINICS IN PRIMARY CARE}

DMG Halpin, S Holmes, J Calvert, D Mclnerney; NHS SW, Taunton, UK

\subsection{6/thoraxjnl-2013-204457.430}

Over $40 \%$ of people with COPD remain undiagnosed. Comorbidities are common in people with COPD but COPD is also a comorbidity of other long term conditions. As SHA respiratory leads in the SW we used this fact to develop a pilot programme to case find people with COPD among patients attending other long term clinics in primary care. The project was developed by the Leads and partially supported by Astra Zeneca, Boehringer Ingelheim, Chiesi \& Novartis who provided support for additional health care professional time, but it was also adopted by Bristol CCG.

Practices were asked to show all current or ex-smokers aged $\geq 35$ attending a long term condition clinic who were not known to have COPD a Patient Information Sheet and ask them to complete a questionnaire designed to help identify people with COPD (1). Patients were also asked to perform microspirometry using an ASMA-1 device, their age, height, \& smoking status were recorded. If they scored highly on the questionnaire and had an FEV1 below the lower limit of normal (LLN), their MRC breathlessness and CAT scores were recorded and they were offered further assessment within the practice to confirm the diagnosis. Smokers were offered referral to cessation services.

573 patients (323 men) were seen in 11 practices between Sep 2012 \& May 2013. Mean age 64.6 (range 36-90). 265 had high questionnaire scores and 115 of these also had FEV1 less than LLN-20\% of all patients seen. The mean FEV1 (\%pred) in these patients was 58.6 (range 5.5-77.6). 86 had an FEV 50$80 \%$ predicted, $2530-50 \%$ predicted. 44 had an MRC score of 0,13 of 1,44 of 2, 8 of 3 and 4 of 5. The mean CAT score was 